\title{
Un moment de la phénoménologie en France : Dufrenne, Lyotard, et le problème de l'expression
}

\author{
Par DANIEL GIOVANNANGELI \\ Université de Liège
}

Le syntagme « le problème de l'expression » figure dans un article de JeanFrançois Lyotard, «À la place de l'homme, l'expression »" . Cet article me retiendra ici parce qu'il se trouve au point d'hérésie entre phénoménologie et structuralisme. Son intérêt excède largement ce qu'il semble être à première vue: un compte rendu analytique du livre de Mikel Dufrenne, Pour l'homme ${ }^{2}$. On ne doit pas sous-estimer l'importance que présenta ce livre. Violemment attaqué - dans sa chronique de télévision du Nouvel observateur du 3 avril 1968 - par Maurice Clavel, alors vulgarisateur inspiré des Mots et les choses, puis, en 1985, évoqué comme l'un de leurs précurseurs par les auteurs de La pensée $68^{3}$, le livre de Dufrenne témoignait d'une résistance phénoménologique de l'humanisme — sartrien mais aussi merleau-pontien - à la montée des structuralismes.

D'une manière, dans son article, Lyotard prenait acte et s'inscrivait lui-même, d'abord et provisoirement, à l'intérieur de cet horizon phénoménologique. Mais, le déplacement n'est pas anodin, il corrigeait le recours dufrennien à l'humanisme en lui substituant une philosophie de l'expression. Ce faisant, Lyotard compliquait le conflit entre la philosophie transcendantale d'inspiration phénoménologique et le courant de pensée d'inspiration structuraliste, esquissant un dépassement qu'allait concrétiser Discours, figure, sa thèse de doctorat, réalisée sous la direction de Dufrenne et publiée

\footnotetext{
1 J.-F. Lyotard, « À la place de l'homme, l'expression », Esprit, juillet-août 1969, p. $155-178$.

${ }^{2}$ M. Dufrenne, Pour l'homme, Paris, Seuil, 1969.

${ }^{3}$ Cf. M. Clavel, Ce que je crois, Paris, Grasset, 1975, p. 134-138; L. Ferry et A. Renaut, La pensée 68. Essai sur l'anti-humanisme contemporain, Paris, Gallimard, 1985, p. 18 , note 1 .
} 
en 1971 dans la «Collection d'esthétique » dirigée par ce dernier ${ }^{1}$. Il n'est pas inutile de signaler, sans pouvoir s'y arrêter ici davantage, que le débat engagé par le compte rendu de Lyotard allait se poursuivre : notamment dans la contribution de Dufrenne au numéro de L'Arc consacré à Lyotard, puis dans son livre Subversion, perversion, ainsi que dans l'allusion critique que dans ses entretiens d'Au juste, Lyotard fait à un autre livre de Dufrenne, Art et Politique ${ }^{2}$.

\section{Lyotard lecteur de Dufrenne}

La discussion se déploie autour du débat que Pour l'homme engage avec l'anthropologie de Claude Lévi-Strauss. Dans la seconde partie, qui en constitue la partie constructive, l'article de Lyotard resserre la problématique de Pour l'homme autour des pages de Dufrenne centrées sur l'article que luimême, Lyotard, avait consacré à Lévi-Strauss ${ }^{3}$. Il cherche à y montrer que la confusion qu'entraîne le titre Pour l'homme s'y résorbe dans une philosophie de la nature et de son expressivité. Autrement dit, qu'en réalité la part désormais prise dans l'ouvrage de Dufrenne par l'humanisme, et surtout par l'humanisme hérité de Sartre, laisse transparaître, en contrebande, une philosophie de la nature qui fait de l'homme un simple lieu et nullement une source, et qui s'accorde, non sans quelque infidélité, avec Merleau-Ponty bien plutôt qu'avec Sartre.

Il reste que cette philosophie de la nature que souligne Lyotard et qui, selon lui, vient contester l'humanisme de surface affirmé par Dufrenne et laisse percer une philosophie de l'expression, souffrirait d'une insuffisance conceptuelle que Lyotard pointe et que la conjonction de Frege et Benveniste permettrait de corriger sur ce point.

«Ne vous trompez pas au titre », avertit Lyotard. C'est que Dufrenne lui-même se tromperait sur l'apport de son propre livre. Ce diagnostic, Lyotard l'assène dès l'entame de son article, « À la place de l'homme, l'expression ». Puis il continue, avec et contre Dufrenne: "Écrire pour l'homme, c'est forcément écrire en ses lieu et place, qui sont vides. Je

${ }^{1}$ J.-F. Lyotard, Discours, figure, Paris, Klincksieck, 1985.

2 Cf. M. Dufrenne, «Doutes sur la "libidiné" ", L'Arc (64), 1976, p. 13-27; Subversion/perversion, Paris, PUF, 1977 ; J.-F. Lyotard et J.-L.Thébaud, Au juste. Conversations, Paris, Christian Bourgois, 1979, p. 170-171.

${ }^{3}$ Cf. J.-F. Lyotard, «Les Indiens ne cueillent pas les fleurs», Annales (20, 1), 1965, p. $62-83$. 
voudrais montrer que, compris ainsi, le plaidoyer de Dufrenne, même si l'auteur parfois paraît s'y tromper, ne fait pas l'éloge de l'humanisme, qui est passé, mais qu'il entrouvre une porte sur un naturalisme de l'expression $»^{1}$. Plus loin, Lyotard écrira aussi que "pour l'homme», signifie «pour le tableau $»^{2}$, puis "pour la physis $»^{3}$. Je ne scruterai pas ces glissements sémantiques. Suggérés dans l'article sur Dufrenne, s'inscrivant manifestement dans le sillage de Merleau-Ponty, ils demanderaient à être prolongés plus que je ne le ferai, par un examen minutieusement intégral de Discours, figure.

Une précaution, peut-être. Quand Dufrenne, repris par Lyotard, use du concept d'expression, il ne peut s'agir d'identifier cette notion, du moins sans autre forme de procès, avec celle que Jacques Derrida a proposée en 1967 dans La voix et le phénomène pour rendre compte du concept husserlien d'Ausdruck en sa distinction essentielle d'avec l'indice (Anzeichen). Par expression, Dufrenne, faisant écho à la phénoménologie du langage de Maurice Merleau-Ponty, entend - il l'écrira lapidairement dans un article consacré à Discours, figure - le "sens donné à même le sensible » ${ }^{4}$. Il faut citer longuement une page de Pour l'homme qui noue l'usage poétique du langage - les mots " rendus à leur nature » — et l'expression de la nature elle-même :

Il arrive que l'outil, au lieu de s'effacer, cherche à valoir par lui-même, exhibant sa propre chair, la matière même du signe, dont le pouvoir signifiant n'est plus alors porté par son insertion dans le système et sa nature diacritique, mais par la vertu même de ses qualités sensibles ${ }^{5}$.

Tout se passe alors comme si l'arbitraire du signe était dépassé, comme si la distance entre le signifiant et le signifié s'annulait :

En nous laissant courir sur l'erre du comme si - en entrant dans la métaphysique - , nous en viendrions à dire que, lorsque les mots par la poésie sont mis en liberté et rendus à leur nature, c'est la nature qui s'exprime, c'est elle qui veut le langage pour se dire à travers la parole poétique ${ }^{6}$.

${ }^{1}$ J.-F. Lyotard, « À la place de l'homme, l'expression », art.cit., p. 155.

${ }^{2}$ Ibid., p. 156.

${ }^{3}$ Ibid., p. 156.

${ }^{4}$ M. Dufrenne, « Doutes sur la "libidiné" », art. cit., p. 14.

${ }^{5}$ M. Dufrenne, Pour l'homme, op. cit., p. 164.

${ }^{6}$ Ibid., p. 164. 
Rapporté à la Phénoménologie de l'expérience esthétique (1953), le livre de 1968, Pour l'homme, fait une place accrue et accueillante à la pensée sartrienne. Expressément, Dufrenne entend y conjuguer Sartre avec MerleauPonty ${ }^{1}$, tandis que son livre de 1953 opposait le poids du sensible à la déréalisation qui caractérise l'imaginaire sartrien. En 1963, Le poétique allait marquer un net infléchissement vers une philosophie de la nature, en accentuant le rapprochement avec la pensée de Merleau-Ponty. Si, d'un côté, l'héritage de la philosophie critique et de la réduction phénoménologique continue de soutenir la réflexion de Pour l'homme, d'un autre côté, Dufrenne s'y montre de plus en plus occupé de ce que Lyotard désigne comme " l'“avant" de la séparation critique et phénoménologique que manifestent le poème, le tableau, le lieu naturel $»^{2}$. En d'autres termes, Dufrenne s'y montre soucieux de la présence antérieure à l'objectivation, de la connaturalité antérieure à la scission du sujet et de l'objet.

La question que cette phénoménologie appelle est alors pour Lyotard la suivante : ce lieu, pourquoi Dufrenne le nomme-t-il « homme»? Cet homme, en tout cas, est sans commune mesure avec l'homme de l'humanisme. Coïncidant avec le lieu où la nature trouve à s'actualiser, où c'est elle qui exige «son dépassement ou sa venue à soi »", coïncidant avec l'expression de la nature, l'homme que cerne Dufrenne est très éloigné d'un humanisme. Ou plutôt, l'humanisme que délivre Dufrenne est en ultime instance la manifestation d'un "naturalisme achevé»". Si Dufrenne réhabilite l'homme, c'est en tant que l'homme s'identifie au " lieu où s'opère le contact et la séparation de la nature avec elle-même ${ }^{5}$. Ainsi compris comme sujet au cœur de la scission d'une part (Sartre), nostalgique d'autre part de l'unité (Merleau-Ponty), l'homme est le nom que Dufrenne donne,

${ }^{1}$ Dufrenne parle lui-même, par exemple, de « conjuguer les langages de Sartre et de Merleau-Ponty : de mêler à la description du comportement volontaire l'eidétique du soi, et somme tout d'identifier le psychologique et le transcendantal» (Ibid., p. 187, note 14). Entre Sartre et Merleau-Ponty, il dit se refuser à un « choix radical». À ses yeux, "Merleau-Ponty a raison en ce qu'il nous ramène près de l'origine et nous invite à penser le monisme. Sartre a raison en ce qu'il nous considère dans le présent et nous invite à penser le dualisme ». La clé de cette conjugaison réside dans la philosophie de la nature : «Ne pouvons-nous être à la fois poètes de l'origine et artisans de l'histoire, assumant ce statut ambigu d'un être qui appartient à la nature et que la nature veut séparé ?» (Ibid., p. 149, note 3).

${ }^{2}$ J.-F. Lyotard, «À la place de l'homme, l'expression », art. cit., p. 157.

${ }^{3}$ Ibid., p. 159.

${ }^{4}$ Ibid., p. 160

${ }^{5}$ Ibid., p. 158. 
plutôt malencontreusement, à ce lieu où la nature s'exprime, se sépare d'ellemême et se réfléchit « d'une réflexion préréflexive » ${ }^{1}$.

Passant en revue les diverses modalités de la question dont l'homme est pour Dufrenne le nom ${ }^{2}$, Lyotard revient sur l'idée d'une «culture lourde» que soulevait sa propre étude de La pensée sauvage. Une place importante est en effet ménagée par l'auteur de Pour l'homme à cette analyse dans laquelle Lyotard faisait entendre le refus du sauvage (au sens lévistraussien) à s'éprouver comme, écrit Dufrenne, «le lieu anonyme où la structure règne $»^{3}$. De Lyotard lecteur de La pensée sauvage, Dufrenne retient ainsi la conception de la culture comme ce en quoi le sens - j'y reviendrai avec insistance - est immanent à tous les sensibles. La lecture critique de Lévi-Strauss amènerait Lyotard à souligner que la culture sauvage est une sorte de "religio pratique » où " chaque activité est lourde de sens », où «les objets sont», selon la formule de Lévi-Strauss (à Georges Charbonnier) que reprend Lyotard, «plus lourds, plus denses, [...] chargés d'une quantité de choses dont nous avons appris à les purger »; la culture sauvage « transcrit le soleil en danse, l'ancêtre en ours, le serpent en phallus, le ciel et la terre en village ${ }^{4}$. De ce commentaire de Lyotard jugé «très remarquable $»^{5}$ par Dufrenne, Pour l'homme retient la quasi-simultanéité de la nature et de la culture, la déhiscence intime de l'une en l'autre, leur identité mais aussi leur différence. «Si spontanément vécue qu'elle soit » ${ }^{6}$, la culture demeure seconde et exige un apprentissage qui culmine et s'accomplit dans l'initiation : Lyotard et Dufrenne s'accordent là-dessus. Si, montrait l'article de Lyotard sur Lévi-Strauss, sa lourdeur rapproche la culture sauvage du corps vivant, elle «diffère néanmoins du contrat originaire que ce dernier entretient avec le monde $»^{7}$. La culture, à la différence du corps, ne reste pas immergée dans le sensible signifiant. Elle s'en écarte, ne serait-ce qu'à peine mais décisivement, comme en témoigne cette seconde nature que Bourdieu thématisera dans le sillage de Marcel Mauss et de Merleau-Ponty :

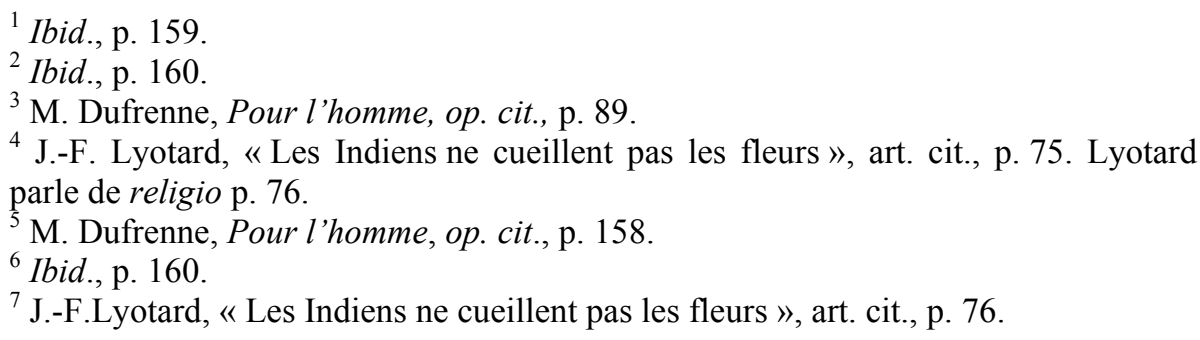


Nous avons de cela un témoignage irrécusable : le sens du haut et du bas, du loin et du près, du vert et du jaune ne s'apprend pas, il s'établit en même temps que le corps comme structure des correspondances sensibles se parachève. Mais le sens de la lune, de l'aigle, de l'épouse et du cuivre s'institue comme une langue maternelle s'apprend; et l'apprentissage trouve consécration dans l'initiation. Tandis que le corps n'a pas de début pour luimême, il y a un commencement de la « seconde nature $»{ }^{1}$.

Cette proximité déclarée de Dufrenne à Lyotard ne doit cependant pas être exagérée. Pour Dufrenne, "sous sa forme originaire, la culture est presque contemporaine de la nature $»^{2}$. Semblablement, comme la culture est presque de la nature, le langage, sous sa forme originaire - l'originarité, ajouterai-je, de la parole parlante dégagée par Merleau-Ponty —, serait presque de la nature :

Il faudrait en dire autant du langage, qu'il faut bien évoquer sitôt qu'on parle de la culture, puisqu'il en est à la fois une partie et la condition. Lui aussi est naturel, ou plus exactement se situe à une distance minimale de la nature : cette même distance qu'il faut assigner à la culture, et qui signifie à l'homme dans le monde son destin de corrélat du monde, sa vocation d'être séparé qu'il accomplira aussi bien en inventant la pensée formelle que la civilisation mécanique ${ }^{3}$.

Mais si Dufrenne se fait ici l'écho de l'analyse de Lyotard, l'interprétation qu'il en dégage reste prise dans une conception phénoménologique du langage dont Lyotard s'écarte. Il n'est pas anodin que, tandis que Lyotard parle de désignation («Ainsi la culture sauvage désigne beaucoup plus que ce qu'elle nomme »), Dufrenne parle de signifié :

L'homme reste au monde en s'en séparant, parce que la pensée reste incarnée, parce que le monde appelle ce langage où il trouve son substitut, parce que le signifié reste attaché aux signes qui s'en distinguent pour le signifier ${ }^{4}$.

Quand Dufrenne fait ainsi sienne, après Lyotard, l'idée de culture lourde, il témoignerait surtout, suivant ce dernier, que l'homme n'est pas le foyer de la Sinngebung. Plus qu'à Sartre, la référence à Merleau-Ponty resterait prégnante. Non, il est vrai, sans gauchissement. La pensée de Dufrenne serait

\footnotetext{
${ }^{1}$ Ibid., p. 77.

${ }^{2}$ M. Dufrenne, Pour l'homme, op. cit., p. 159.

${ }^{3}$ Ibid., p. 163.

${ }^{4}$ Ibid., p. 165.
} 
menacée par la confusion entre ce qui est de l'ordre du transcendantal et ce qui ressortit à l'épistémologie ${ }^{1}$. Son infidélité aux précautions que prenait la Phénoménologie de la perception à séparer critique et phénoménologie d'avec la science est à cet égard flagrante.

Il demeure qu'en plus profonde analyse, la référence à Merleau-Ponty ne suffira plus à Lyotard. Et que, si féconde qu'elle soit pour une philosophie de la nature, il n'est pas sûr qu'elle satisfasse à la constitution d'une philosophie de l'expression. La dépendance de Dufrenne à l'égard de la phénoménologie du langage dans sa version merleau-pontienne empêche de faire droit à ce qui sépare et distingue le voir et le dire. Sur ce point, Hegel avait décisivement montré que "d'une certaine manière le langage ne peut pas rejoindre le sensible et l'absorber $»^{2}$. Quand Dufrenne s'autorise de Merleau-Ponty pour faire de l'expressif ce qui fonde en le précédant le discours, il opte pour la continuité entre nature et langage que, s'autorisant, lui, de Hegel, Lyotard récuse. L'erreur du structuralisme aura sans doute été de mettre "partout de la parole», et, de la sorte, d' " écraser l'écart entre exprimer et dire $»^{3}$. Mais quand, de son côté, Dufrenne inscrit sa métaphysique de la nature au point de la plus grande proximité entre Sartre et Merleau-Ponty ${ }^{4}$, ne manque-t-il pas ce qui différencie essentiellement le voir et le dire ?

C'est vainement que Dufrenne s'emploie à considérer «le transcendantal du langage $»^{5}$ sur le modèle de la perception. Plus précisément,

1 Je ne reviendrai pas ici, pour l'avoir fait ailleurs depuis longtemps, sur la signification à un certain degré originale que Dufrenne confère aux philosophèmes d'a priori matériel et de transcendantal. En témoigne, parmi cent autres passages, cette réflexion (de 1962, autour de Merleau-Ponty) : «Oui, le monde m'est toujours donné. [...] Est-ce à dire que ma présence soit passive ? Non : il faut que je recueille ce sens qui s'ordonne à moi, et l'idée d'un monde sans l'homme est finalement une idée vaine. [ ...] Ce qui est premier — disons transcendantal —, c'est donc bien l'apparaître, l'émergence du sens dans la présence » (M. Dufrenne, Jalons, La Haye, M. Nijhoff, 1966, p. 212).

${ }^{2}$ J.-F. Lyotard, «À la place de l'homme, l'expression », art. cit., p. 173.

${ }^{3}$ J.-F. Lyotard, « Les Indiens ne cueillent pas les fleurs », art. cit., p. 67.

${ }^{4}$ « On peut dire avec Sartre que la mobilité est ce qui, jusque dans l'en-soi, annonce ce que Merleau-Ponty appelle recroisement, on peut dire avec Husserl et avec le premier Merleau-Ponty que le temps, parce qu'il est la synthèse passive originaire, est déjà à lui seul l'absolu de la manifestation, on peut dire avec Dufrenne que la nature a déjà en soi le pouvoir de se mirer, une négativité suffisante pour que naisse le regard. Mais tout cela fait le voir, non le dire.» (J.-F. Lyotard, "À la place de l'homme, l'expression ", art. cit., p. 172-173).

${ }^{5}$ Ibid., p. 175. 
lorsqu'il analyse signification et expression, la perspective phénoménologique qui est la sienne dégage l'essence du voir, non celle du dire. La question que soulève Lyotard est provoquée par deux formules qu'enchaîne Dufrenne dans Le poétique (1963). Quel sens peut-il y avoir à parler, comme Dufrenne le fait là, d' « un langage de la nature» et d'écrire que «dans l'expression, c'est la nature qui s'exprime » ? La réponse est contrastée. Elle consiste d'abord à refuser la première formule, ou du moins à ne lui reconnaître de pertinence que métaphorique. Elle admet ensuite la seconde formule ; mais c'est pour aussitôt en marquer les limites.

\section{Critique de l'expression selon Dufrenne}

Il importe en effet à Lyotard de le souligner, cette parole que Dufrenne prête à la nature, cette expressivité qui la distingue de l'en-soi sartrien, est différente de " celle dans laquelle elle s'exprime ${ }^{1}$. Strictement considéré, le langage est, dans la perspective husserlienne, un objet idéal. Aussi convientil de remarquer qu'en rigueur, la nature ne parle pas. L'erreur de MerleauPonty, dont hériterait Dufrenne, aura été de rapporter le langage au modèle perceptuel. Et il est abusif de s'en remettre à l'analogie qui poserait que "dans l'ordre linguistique le geste de parole soit au système de langue comme le geste de voir est au système physico-physiologique de la vision dans l'ordre perceptif $»^{2}$. Avant que «le geste parlant» du locuteur ne sélectionne des unités dans le système virtuel de la langue, ces unités comportaient déjà une signification. Mais il en va autrement du voir. L'ordre perceptif

ne recèle aucune signifiance avant le sentir : l'œil optique ou anatomique ne voit pas, il n'est pas un œil sensible; l'ordre du langage est au contraire dépositaire de significations avant que le geste parlant y sélectionne et y enchaîne des unités pour y tracer un sens. Avant ce sens, il y avait de la signification, latente dans le système virtuel où puise le locuteur; ici l'acte baigne dans le même milieu sémantique que le système.

Tout autre est la perception :

Avant le sensible, il n'y a rien que des conditions, insensibles, supposées et construites, les conditions physico-physiologiques qui rendent possible la

${ }^{1}$ Ibid., p. 172.

${ }^{2}$ Ibid., p. 176. 
perception. Avec l'œil s'érige le monde des sensibles; avec la parole, le monde des significations ne s'érige pas, il se communique ; à la rigueur, il se déforme, par transgression ${ }^{1}$.

Hegel a dégagé au commencement de la Phénoménologie de l'esprit cette indicibilité de l'indiquer, ce silence que le discours suppose comme sa référence :

Il y a un silence naturel qui occupe la parole, ce silence est celui d'indiquer, aufzeigen, et il reste indicible dès le commencement de la Phénoménologie de l'esprit; on voit bien, dans Hegel, qu'entre le langage et le sensible (la nature), le passage ne se fait pas, qu'il y a à la fois plus et moins dans le dire que dans le voir ${ }^{2}$.

Ainsi, ce qui ferait défaut à la théorie de l'expression - insistons : l'expression, « ce sens donné à même le sensible » — que Dufrenne élabore, c'est de distinguer rigoureusement désignation et signification. Quand Dufrenne, soulignant à nouveau l'immanence du signifié au signifiant, écrit dans Le poétique: «Dire que le mot porte en lui son sens [...], c'est dire qu'il ne désigne pas autre chose», Lyotard corrige. "On ne peut dire que «le mot [...] ne désigne pas autre chose». Mais il est légitime de «dire qu'il ne signifie pas autre chose $» »^{3}$. Il aura manqué à Dufrenne lorsqu'il formule le problème de l'expression, de distinguer les deux axes du discours. Ce dont souffre son analyse, c'est de ne pas dissocier, "comme Frege et aussi Benveniste le requièrent, deux dimensions croisées sur le discours: la signification (Sinn), la désignation (Bedeutung) $\gg^{4}$.

En somme, à tort, Dufrenne croit défendre un humanisme alors qu'il fait droit à une philosophie de l'expression. Et à tort, il confond l'expression de la nature avec le langage. Or, ce qui caractérise l'expression ne se comprend que dans son extériorité et son altérité à la signification linguistique. Pour supporter l'idée selon laquelle la nature parle, Dufrenne en

${ }^{1}$ Ibid., p. 176.

2 Ibid., p. 173. Dans son livre de 1954 sur La phénoménologie, Lyotard nuançait l'idée suivant laquelle entre le rationalisme hégélien et Husserl «le conflit est total ». Il suggérait que «si l'on considère que l'entreprise phénoménologique est fondamentalement contradictoire en tant que désignation par le langage d'un signifié pré-logique dans l'être, elle est inachevée à jamais parce que renvoyée dialectiquement de l'être au sens à travers l'analyse intentionnelle » et que, dès lors, «la vérité est devenir » (La phénoménologie, Paris, PUF, $6^{\mathrm{e}}$ éd., 1967, p. 45-46).

${ }^{3}$ Ibid., p. 172.

${ }^{4}$ Ibid., p. 172-173. 
appelle aux enseignements de la linguistique et plus précisément, il renvoie à ce que Roman Jakobson a cerné comme la fonction poétique du langage, que caractérise « l'accent mis sur le message pour son propre compte ${ }^{1}$. " C'est l'élision de la fonction référentielle, dans la terminologie de Jakobson. Dans celle de Dufrenne, c'est : la nature qui parle $»^{2}$. Or, de l'avis de Lyotard, l'élision de la fonction référentielle qui caractérise la fonction poétique n'équivaut pas à ce que Dufrenne cerne depuis sa Phénoménologie de l'expérience esthétique (1953) comme signe index sui : la peinture abstraite continue de signifier, y écrivait Dufrenne, l'objet esthétique est un signe, mais il «n'est pas un signe qui renvoie à autre chose qu'à lui-même " ${ }^{3}$. Commentant Husserl, Derrida remarque qu'il revient probablement au même d'être un signe index sui et de n'être pas un signe ${ }^{4}$. Lyotard, lui, objecte que dans le langage expressif, l'immanence n'est pas, comme le pense Dufrenne, celle du signifié au signifiant. Il s'y agit plus exactement de l'immanence du désigné au signe :

Pour qu'il y ait expression, il faut qu'il y ait une épaisseur, un devant et un derrière entre lesquels le $e x$ - se joue. Cette épaisseur est de constitution : le signe est là pour autre chose, qu'il manifeste et cache à la fois. Cette distance qui se tend pour se faire épaisseur est le secret de la transcendance $[\ldots]^{5}$.

Parler d'immanence du désigné au signe, c'est corriger l'immanence du signifié au signifiant. C'est, autrement dit, faire droit à la transcendance du désigné, excéder la signification dans la désignation. Déjà le commentaire de La pensée sauvage avait dégagé ce statut ambigu du signe. Lyotard y distinguait entre la structure sémiologique du corps, où « un signe remplace l'autre », et celle de la culture, où un "signe appelle l'autre et l'accueille sans lui-même s'effacer » :

Transcrire telle couleur en contraction des muscles extenseurs est dire la vibration lumineuse dans le registre du tonus musculaire; un signe remplace l'autre. Traduire l'homme en serpent et le serpent en pluie ne fait pas oublier que l'homme est homme, le serpent reptile, la pluie eau du ciel. La distance

${ }^{1}$ R. Jakobson, Essais de linguistique générale, tr. fr. N. Ruwet, Paris, Minuit, 1963, p. 218.

${ }^{2}$ J.-F. Lyotard, « À la place de l'homme, l'expression », art. cit., p. 172.

${ }^{3}$ M. Dufrenne, Phénoménologie de l'expérience esthétique, Paris, PUF, $2^{\mathrm{e}}$ éd. 1967, p. 166.

${ }^{4}$ J. Derrida, La voix et le phénomène, Paris, PUF, $2^{\mathrm{e}}$ éd. corrigée, 1998, p. 66, note 1.

${ }^{5}$ J.-F. Lyotard, «À la place de l'homme, l'expression », art. cit., p. 172. 
d'un signe à l'autre est conservée dans leur unité, comme quand nous disons d'un homme influençable : c'est un caméléon ${ }^{1}$.

\section{De la critique de Dufrenne à la critique de Derrida}

Il resterait, pour faire bonne mesure, à considérer l'accueil que Dufrenne réservera ensuite à Lyotard. L'article de L'Arc commence, dans un premier temps, par concéder à Lyotard qu'il n'est pas de désignation sans signification :

Lyrique ou savant, le discours exploite toujours le caractère référentiel du langage : le langage mord sur le monde, et la parole s'en recommande, car on parle pour dire quelque chose. Le langage ne se referme pas sur lui-même. Il est vrai pourtant que la langue doit être un système clos pour que s'y constituent des significations. Il est vrai que la désignation n'est possible qu'associée à la signification ${ }^{2}$.

Ce serait donc faire tort à la pensée de Dufrenne que d'ignorer - Lyotard ne le fait du reste nullement - la place qu'elle réserve aux a priori matériels qui informent l'expérience. Ainsi, dans un article de 1967, Dufrenne évoquait-il d'un trait la distinction opérée par Frege et par Husserl, entre Sinn et Bedeutung. Husserl, rappelait-il, distingue entre le sens propre à la morphologie, que définit son opposition au non-sens ( «homme donc oui »), le sens propre au jugement distinct, qui s'oppose au contre-sens (« tout S est $\mathrm{P}$, sauf quelques $\mathrm{P} »)$ et enfin un sens reposant sur «l'homogénéité matérielle des noyaux » et qui s'oppose au faux-sens (Dufrenne emprunte à Blanché cet exemple: « 3 est ovipare »). Et il remarquait que tandis que «les deux premières acceptions définissent le sens par la grammaire, par l'accord avec les règles d'emploi et de manipulation des termes ${ }^{3}$, c'est l'adéquation au contenu du jugement qui définit la troisième acception. Pour l'homme accomplit un pas de plus. Dufrenne y souligne le choix des exemples de contresens qu'invoque la $4^{\mathrm{e}}$ des Recherches logiques. Non pas des énoncés formels comme, dans Logique formelle et logique transcendantale, " tous les A sont B, parmi lesquels quelques-uns ne sont pas B », mais des «énoncés matériels » comme « du fer en bois ». Et Dufrenne y avance l'hypothèse que

\footnotetext{
${ }^{1}$ J.-F. Lyotard, « Les Indiens ne cueillent pas les fleurs », art. cit., p. 77.

${ }^{2}$ M. Dufrenne, «Doutes sur la "libidiné" », art. cit., p. 16.

${ }^{3}$ M. Dufrenne, «La critique littéraire : structure et sens », repris dans Esthétique et philosophie, Paris, Klincksieck, 1967, p. 131.
} 
cela pourrait manifester que «le contresens où la pensée se contredit contredit le réel parce que la loi qui est reniée est une loi du réel $»^{1}$.

Rien d'étonnant dès lors si, dans L'Arc, Dufrenne reprend tranquillement à son compte le partage et l'articulation entre signification et désignation que Lyotard lui opposait. Par contre, la critique que Lyotard esquissait vaudra plus sévèrement, en dépit de l'admiration déclarée, pour Derrida. La longue note qui achève le chapitre de Discours, figure consacré à lire l'article de Frege, Über Sinn und Bedeutung (1892), souligne les bornes de la phénoménologie husserlienne. Et elle adresse à Jacques Derrida le reproche de rester sous l'emprise de la prémisse husserlienne. Je me reporte à cette longue note serrée ${ }^{2}$. Il n'y est pas question de Dufrenne, évoqué ailleurs dans le livre, mais la convergence entre Frege et Benveniste allusivement dressée contre Dufrenne, s'y trouve explicitée. En réalité, cette note comporte une critique de la solution au problème du déictique que Derrida développe dans La voix et le phénomène. Derrida y est en somme accusé de se faire plus husserlien que Husserl en retournant la prémisse husserlienne contre Husserl.

On ne peut identifier la signification selon Frege d'une part, selon Husserl d'autre part. Certes, alors que le début de la $1^{\text {re }}$ des Recherches logiques refusait de distinguer Sinn et Bedeutung comme contraire à l'usage, le $\S 4$ prenait pourtant acte de cette distinction. Il n'empêche que si l'un et l'autre conçoivent la signification sur le mode de l'objectivité (Husserl le fait dans la $1^{\text {re }}$ des Recherches logiques),

le premier la constitue au moyen d'une opération (l'épreuve de commutation) qui permet de fixer les intervalles séparant les termes et produisant l'effet de sens, tandis que le second la pose comme un "vouloir-dire" virtuel qui sera actualisé et animé par la « vie » d'un sujet cherchant l'intuition ${ }^{3}$.

Et quoiqu'il soit possible de rapprocher l'épreuve de la commutation (« quand ai-je le droit de remplacer $a$ par $b$ ? » $»^{4}$ ) et la variation imaginaire dans la mesure où toutes deux accomplissent un geste qui transgresse l'immédiateté, «chez Frege, le résultat de cet acte, le concept, se définit seulement par une identité du type $\mathrm{a}=\mathrm{b}$; au contraire, l'essence husserlienne est une signification saisie "en personne" par une intuition positive de

\footnotetext{
${ }^{1}$ M. Dufrenne, Pour l'homme, op. cit., p. 177.

2 J.-F. Lyotard, Discours, figure, op. cit., note 18, p. 115-116.

3 J.-F. Lyotard, Discours, figure, op. cit., p. 115-116.

${ }^{4}$ Ibid., p. 112. «C'est le problème du jugement synthétique, mais il est abordé en termes de sémiologie et non plus de criticisme $[\ldots] »($ p. 110).
}

335 
l'Ego $»^{1}$. En somme, alors que le geste de Frege s'inscrit dans l'horizon leibnizien en comprenant la signification " en termes de système », celui de Husserl s'inscrit dans l'horizon cartésien de l'intuitus.

La même présupposition commande au statut du Je dans la $1^{\text {re }}$ des Recherches logiques, celle-là que Derrida affronte précisément dans La voix et le phénomène. Selon Lyotard, Benveniste et Frege convergent sur ce point. En distinguant Sinn et Bedeutung, Frege montrait que la différence qui les sépare ne réside pas dans l'objectivité, mais qu'elle est ailleurs. Tandis que l'un est de l'ordre du système, l'autre est extérieure à celui-ci :

Frege distingue la lune (Bedeutung), visée dans l'objectif d'une lunette astronomique, et son image (Sinn) située dans le système optique de cette lunette. La comparaison dit clairement que la lune n'est pas plus objective que l'image, que l'image n'est pas moins objective que la lune, et que la seule différence pertinente réside en ce que l'une est dans le système (optique, et analogiquement linguistique) et l'autre au dehors ${ }^{2}$.

Quant à Benveniste, dans les années cinquante, il allait montrer que le signifiant déictique possède un statut qui le distingue radicalement des autres signifiants du système et qu'il s'impose d'« en référer l'usage à une extériorité supposée ». Autrement dit en conjuguant la terminologie de Frege avec celle de Benveniste, "sans cette dimension de désignation, aucun déictique n'est concevable $»^{3}$. Husserl, par contre, continue de parler, dans la $1^{\mathrm{re}}$ des Recherches logiques du moins, de «la signification du Je»" Sur ce point précis, Lyotard prend ses distances à l'égard de ce qu'il appelle « la critique remarquable $»^{5}$ développée en 1967 par La voix et le phénomène.

Il est vrai que Derrida joue là Husserl contre Husserl. Quand Husserl écrit, par exemple, que «le mot $J e$ nomme, suivant les cas, une personne

${ }^{1}$ Ibid., p. 115. La convergence entre la transcendance selon Frege et selon Husserl est cernée p. 110.

${ }^{2}$ Ibid., p. 115.

${ }^{3}$ Ibid., p. 116. Je note d'un mot que Pour l'homme (op. cit., p. 218) se fait l'écho de Paul Ricœur qui saluait la rencontre entre ce qu'il appelait «la phénoménologie du sujet parlant» et les recherches de Benveniste sur «les formes de l'allocution inhérentes à l'instance de discours ».

${ }^{4} C f$., par ex., E. Husserl, Recherches logiques, t. 2, tr. fr. H. Élie, A.L. Kelkel et R. Schérer, Paris, PUF, 1961, p. 95 : « Dans le discours solitaire, la signification du je se réalise essentiellement dans la représentation immédiate de notre propre personnalité, et c'est là que réside donc aussi la signification de ce mot dans le discours communicatif ».

${ }^{5}$ J.-F. Lyotard, Discours, figure, op. cit., p. 116. 
différente, et [...] le fait au moyen d'une Bedeutung toujours nouvelle », Derrida s'étonne en effet de le voir contredire l'indépendance qui détache l'intention de l'intuition remplissante. C'est en s'autorisant des prémisses mêmes de Husserl qu'il objecte que « de même que je n'ai pas besoin de percevoir pour comprendre un énoncé de perception, je n'ai pas besoin de l'intuition de l'objet $J e$ pour comprendre le mot $J e »{ }^{1}$. Nous comprenons ce mot même si l'auteur ne nous en est pas connu, lorsqu'il est fictif ou encore - Derrida parle alors de la "valeur structurellement testamentaire ${ }^{2}$ de l'idéalité du mot $J e$ - lorsqu'il est mort. De la sorte, Derrida, inquiet de la présupposition métaphysique de la présence, ramène, dans les termes de Lyotard, "le signifiant déictique au statut de tout autre signifiant du système $»^{3}$. Au fond, il se tromperait de cible. À raison, Derrida dénonce l'inintelligibilité de l'idée de "signification indiquée », qu'il juge contradictoire avec le principe de l'idéalité du sens. Mais, on le voit, cette critique qu'il adresse à Husserl suppose la problématique husserlienne. En quelque sorte, critiquant Husserl, Derrida resterait husserlien et, d'une manière, plus husserlien que Husserl. Il lui eût fallu opter pour Frege et Benveniste en s'écartant de Husserl. À Derrida, Lyotard oppose que « le déictique n'est pas une simple valeur à l'intérieur du système, mais un élément qui de l'intérieur renvoie à l'extérieur; il n'est pas pensable dans le système ; mais à travers lui $»^{4}$.

\section{Retour à Frege ?}

On serait volontiers tenté de parler d'un retour à Frege. Chez ce dernier, Lyotard retrouve en effet ce

point où l'exclusion de la désignation au bénéfice de la signification d'un côté, et de l'autre l'enfouissement de la structure-clef du Sinn sous les analyses intentionnelles, ne sont pas encore consommés, où l'union des deux grands thèmes kantiens du transcendantal comme subjectivité et du transcendantal comme structure n'est pas défaite, mais au contraire élaborée $[\ldots]^{5}$.

${ }^{1}$ J. Derrida, La voix et le phénomène, op. cit., p. 107.

${ }^{2}$ Ibid., p. 107.

3 J.-F. Lyotard, Discours, figure, op. cit., p. 116.

${ }^{4}$ Ibid., p. 116.

${ }^{5}$ Ibid., p. 105. 
Il serait en réalité plus exact de dire que la conceptualité de Frege sert de pierre de touche à Lyotard. Non qu'il s'y arrime ni s'y arrête. La synonymie de l'expression avec la désignation ne suffira pas à épuiser le champ qu'ouvrira en 1971 Discours, figure.

Quand la dialectique étend sa prétention à l'objet, à l'autre du concept qu'est le sensible, alors elle excède sa propre portée, et de savoir se mue en idéologie. On peut bien affirmer que tout est dicible, c'est vrai, mais ce qui ne l'est pas, c'est que la signification du discours recueille tout le sens du dicible. On peut dire que l'arbre est vert, mais on n'aura pas mis la couleur dans la phrase. Or la couleur est du sens. La négativité de signification échoue sur celle de désignation, non pas en ce qu'il y aurait une indicibilité radicale du monde et un destin de silence, mais en ceci qu'à l'effort de signifier, correspond toujours un report symétrique du désigner ${ }^{1}$.

De Frege, Lyotard retient fondamentalement que «tout langage est essentiellement ouvert sur du non-langage $»^{2}$. Ainsi, reconsidérée à la lumière de Frege, la dialectique hégélienne, dont l'article sur Pour l'homme se réclamait encore, apparaît grevée d'une réduction illégitime de la Bedeutung au Sinn.

L'espoir d'enfermer l'objet tout entier dans le discours doit être abandonné si on le nourrit de cette façon-là, et c'est à quoi il faut s'en prendre dans Hegel. En revanche l'espace de désignation habite effectivement le discours, mais en deçà de ce qu'il signifie, dans son expression ${ }^{3}$.

Frege sert de la sorte de contrepoint provisoire à l'ambition hégélienne. Cet espace de désignation, continue Lyotard, « je l'appelle provisoirement espace de désignation parce que ses propriétés semblent analogues à celles de cet espace, et contredisent celles de l'espace linguistique. Leur trait commun est la figure, on l'appellera espace figural $»^{4}$. Ce qui retient provisoirement, stratégiquement, Lyotard, c'est ce qui, de la désignation, tout à la fois suppose la signification comme sa condition de possibilité et excède la signification vers le dehors du système. La désignation s'appuie, sans s'y réduire, sur la négativité diacritique qui fonde la signification. C'est en réactivant sur nouveaux frais l'exigence de figurabilité mise en lumière par Freud, en forgeant le concept de figural comme expression du non-discursif au sein du discours qu'il vient déranger, que Discours, figure trouvera les

${ }^{1}$ Ibid., p. 51-52.

${ }^{2}$ Ibid., p. 108.

${ }^{3}$ Ibid., p. 52.

${ }^{4}$ Ibid., p. 52 ; c'est moi qui souligne. 
ressources d'une reprise inédite de l'expression, ou encore de la désignation, en tant qu'il y va de la transcendance non discursive des choses. 